Journal of Quantitative Spectroscopy \&

Radiative Transfer 75 (2002) 611-624
Journal of

Quantitative

Spectroscopy \&

Radiative

Transfer

www.elsevier.com/locate/jqsrt

\title{
A simple new radiative transfer model for simulating the effect of cirrus clouds in the microwave spectral region
}

\author{
T.R. Sreerekha*, S. Buehler, C. Emde \\ Institute of Environmental Physics, University of Bremen, P.O. Box 330440, D-28334 Bremen, Germany
}

Received 8 January 2002; accepted 12 March 2002

\begin{abstract}
The upwelling atmospheric radiation in the millimeter wave spectral range is influenced by the presence of cirrus clouds. A plane parallel radiative transfer model which can take into account the effect of multiple scattering by ice particles in the cirrus has been developed and is used to simulate the brightness temperatures as they would be measured by a satellite instrument. The model uses an iterative procedure to solve the radiative transfer equation. The formulation of the model is such that it can easily be adapted to treat the full specific intensity vector instead of just the scalar total intensity. A convergence test for the model is explained and two cirrus cloud scenarios are simulated. The results illustrate the linearity of microwave radiative transfer for not too strong cirrus clouds in this frequency region. (C) 2002 Elsevier Science Ltd. All rights reserved.
\end{abstract}

Keywords: Radiative transfer; Scattering; Microwave; Cirrus clouds

\section{Introduction}

Satellite remote sensing data are extensively used by the numerical weather prediction community for global measurements of geophysical parameters. Recently, the mm channels on AMSU-B [1], SSMT-2 [2], etc. are providing global data on the distribution of humidity in the upper troposphere which is currently one of the largest uncertainties. However, in cloudy areas these data are influenced by the presence of cirrus clouds, which have distinct radiative transfer properties compared to other cloud types. Our goal is to eventually improve the utilization of these data by better quantifying the cirrus cloud impact. The radiative transfer (RT) model development is the first essential step towards this goal.

Another important motivation for our work is the recently proposed ESA opportunity mission cloud ice water submillimeter wave imaging radiometer (CIWISR). CIWSIR is mainly based on

\footnotetext{
${ }^{*}$ Corresponding author. Fax: +49-421-218-4555.

E-mail address: rekha@uni-bremen.de (T.R. Sreerekha).
} 
the study of Miao et al. [3] and Evans et al. [4] in which they have discussed the rationale for adopting sub-mm wave channels for retrieving cirrus cloud ice water path (IWP) and characteristic particle size. As the water vapor absorption is relatively strong in the sub-mm frequency range, the lower atmosphere is opaque so that the effect of surface and lower clouds on the upwelling radiation can be negligible. Moreover, at these frequencies ice particles are weak absorbers, which makes the physical temperature of the cirrus clouds unimportant. As a first application of our new model we try to simulate the effect of cirrus IWP at the CIWSIR frequencies.

We are aware of the fact that there has already been a lot of work done in the area of RT models with scattering. For example, Evans et al. [5] have developed a numerical model that solves the polarized radiative transfer equation for a plane parallel vertically inhomogenous scattering atmosphere. In this model the solution method for the multiple scattering aspect of the problem is doubling and adding. Another example is the model described by Czekala et al. [6] where they have implemented a successive order of scattering method for non-spherical particles in a plane parallel atmosphere. However, developing a new model from scratch makes it easier to make modifications and extensions to suit the requirements. The RT model discussed here now calculates only the first component of the Stokes vector for a plane parallel atmosphere. But the model is formulated in such a way that extending it for other Stokes parameters and geometries is rather easy. The RT model can handle the scattering properties of a homogenous cirrus cloud in a plane parallel atmosphere using an iterative approach to solve the radiative transfer equation.

The article is organized as follows. The vector radiative transfer equation is discussed in Section 2. Our approach to solve this equation for scalar case and clear sky case is explained. The simulation set up for the study including observation frequencies, reference atmosphere, cloud assumptions and single scattering properties are explained in Section 3. Results and discussions for clear sky cases and cloudy cases are in Section 4. Here the validity of the plane parallel and linear approximations are tested by comparing with a spherical model for the clear sky condition and another test is done for validating the convergence of the model. Concluding remarks are presented in Section 5.

\section{The RT equation}

\subsection{The general vector form}

The general RT equation for an atmosphere taking into account extinction, emission and scattering is [7]

$$
\mathrm{d} \mathbf{I}(\mathbf{n}) / \mathrm{d} s=-\mathbf{K}(\mathbf{n}) \mathbf{I}(\mathbf{n})+\mathbf{a}(\mathbf{n}) B(T)+\int_{4 \pi} \mathrm{d} \mathbf{n}^{\prime} \mathbf{Y}\left(\mathbf{n}, \mathbf{n}^{\prime}\right) \mathbf{I}\left(\mathbf{n}^{\prime}\right),
$$

where $\mathbf{I}(\mathbf{n})$ is the four component specific intensity vector of multiply scattered radiation propagating in the direction $\mathbf{n}$. The first component of this vector, $I$ gives the total intensity, the second component $Q$ and the third component $U$ give the measure of linear polarization and the fourth component $V$ gives the measure of circular polarization. The pathlength element measured in the direction $\mathbf{n}$ is $\mathrm{d} s$. The extinction of radiation is described by the $4 \times 4$ extinction coefficient matrix $\mathbf{K}(\mathbf{n})$ which includes the extinction due to gas and particles:

$$
\mathbf{K}(\mathbf{n})=\mathbf{K}^{\mathrm{p}}(\mathbf{n})+\mathbf{K}^{\mathrm{g}}(\mathbf{n}) .
$$


$\mathbf{K}^{\mathrm{p}}(\mathbf{n})$ and $\mathbf{K}^{\mathrm{g}}(\mathbf{n})$ are the extinction coefficients for particle and gas, respectively. From Lambert's law, extinction process must be proportional to the amount of matter [8] which gives

$$
\mathbf{K}^{\mathrm{p}}(\mathbf{n})=n^{\mathrm{p}} \mathbf{L}^{\mathrm{p}}(\mathbf{n})
$$

where $n^{\mathrm{p}}$ is the particle number density and $\mathbf{L}^{\mathrm{p}}(\mathbf{n})$ is the extinction cross-section matrix. Here, $\mathbf{K}^{\mathrm{p}}(\mathbf{n})$ has the dimension of $1 /$ length and $\mathbf{L}^{\mathrm{p}}(\mathbf{n})$ has the dimension of area.

The gaseous extinction coefficient is calculated according to

$$
\mathbf{K}^{\mathrm{g}}(\mathbf{n})=\sum_{i} n_{i}^{\mathrm{g}} \mathbf{L}_{i}^{\mathrm{g}}(\mathbf{n})
$$

where $n_{i}^{\mathrm{g}}$ is the number density of the $i$ th gaseous species and $\mathbf{L}_{i}^{\mathrm{g}}(\mathbf{n})$ is the extinction cross-section of the $i$ th gaseous species.

The second term in Eq. (1) is the thermal source term where $\mathbf{a}(\mathbf{n})$ is the absorption coefficient vector and $B(T)$ is the Planck function at temperature $T$. The particle absorption as well as gaseous absorption is included in $\mathbf{a}(\mathbf{n})$ which can be represented as

$$
\mathbf{a}(\mathbf{n})=\mathbf{a}^{\mathrm{p}}(\mathbf{n})+\mathbf{a}^{\mathrm{g}}(\mathbf{n}) .
$$

The particle absorption coefficient is expressed as

$$
\mathbf{a}^{\mathrm{p}}(\mathbf{n})=n^{\mathrm{p}} \mathbf{b}^{\mathrm{p}}(\mathbf{n})
$$

where $\mathbf{b}^{\mathrm{p}}(\mathbf{n})$ is the absorption cross-section vector of the particles. Similarly for the gaseous absorption coefficient,

$$
\mathbf{a}^{\mathrm{g}}(\mathbf{n})=\sum_{i} n_{i}^{\mathrm{g}} \mathbf{b}_{\mathrm{i}}^{\mathrm{g}}(\mathbf{n})
$$

where $\mathbf{b}_{i}^{\mathrm{g}}(\mathbf{n})$ is the absorption cross-section of the $i$ th gaseous species.

The last term in Eq. (1) is the scattering source term. It describes the contribution of radiation illuminating a small volume element from all directions $\mathbf{n}^{\prime}$ and scattered into the direction $\mathbf{n}$. We decided to call $\mathbf{Y}\left(\mathbf{n}, \mathbf{n}^{\prime}\right)$ the scattering efficiency matrix. It can be expressed in terms of the well-known phase matrix $\mathbf{Z}\left(\mathbf{n}, \mathbf{n}^{\prime}\right)$ as

$$
\mathbf{Y}\left(\mathbf{n}, \mathbf{n}^{\prime}\right)=n^{\mathrm{p}} \mathbf{Z}\left(\mathbf{n}, \mathbf{n}^{\prime}\right) \text {. }
$$

The phase matrix gives the efficiency of scattering between directions $\mathbf{n}$ and $\mathbf{n}^{\prime}$. (The name is historic and has nothing to do with the phase of an electromagnetic wave.)

In the work presented here, Eq. (1) is solved iteratively in a plane parallel atmosphere for the scalar case, i.e., only for the first component of the specific intensity vector. The plane parallel approximation holds when the radius of curvature is much larger than the thickness of the layer. Also keeping in mind that solving this equation for $\mathbf{I}(\mathbf{n})$ may cause computational difficulties as it involves the exponential of a matrix, a linear approximation is considered assuming optically thin layers. The advantage of this approach is that it is conceptually simple but fully general and can be extended easily for the full vector equation.

\subsection{The scalar case}

Assuming that all cloud ice particles are spherical in shape and that the incident radiation is unpolarized, the radiative transfer equation for total intensity is written as

$$
\mathrm{d} \mathbf{I}(\mathbf{n}) / \mathrm{d} s=-K_{11}(\mathbf{n}) \mathbf{I}(\mathbf{n})+a_{1}(\mathbf{n}) B(T)+\int_{4 \pi} \mathrm{d} \mathbf{n}^{\prime} Y_{11}\left(\mathbf{n}, \mathbf{n}^{\prime}\right) \mathbf{I}\left(\mathbf{n}^{\prime}\right) .
$$


Here $K_{11}(\mathbf{n})$ is the $(1,1)$ element of the extinction coefficient matrix $\mathbf{K}(\mathbf{n})$ :

$$
\begin{aligned}
& K_{11}(\mathbf{n})=K_{11}^{\mathrm{p}}(\mathbf{n})+K_{11}^{\mathrm{g}}(\mathbf{n}), \\
& K_{11}^{\mathrm{p}}(\mathbf{n})=n^{\mathrm{p}} L_{11}^{\mathrm{p}}(\mathbf{n}) .
\end{aligned}
$$

$L_{11}^{\mathrm{p}}(\mathbf{n})$ is the $(1,1)$ element of the particle extinction cross-section matrix $\mathbf{L}^{\mathrm{p}}(\mathbf{n}) . L_{11}^{\mathrm{p}}(\mathbf{n})$ is calculated using the formula [7]

$$
L_{11}^{\mathrm{p}}(\mathbf{n})=\lambda \operatorname{Im}\left[\left(S_{11}(\mathbf{n})+S_{22}(\mathbf{n})\right)\right],
$$

where $\lambda$ is the wavelength. $S_{11}(\mathbf{n})$ and $S_{22}(\mathbf{n})$ are the elements of the forward scattering amplitude matrix and are calculated using the $T$-matrix algorithm of Mishchenko [9]. For the gaseous extinction,

$$
K_{11}^{\mathrm{g}}(\mathbf{n})=n_{i}^{\mathrm{g}} L_{11, i}^{\mathrm{g}}(\mathbf{n}) .
$$

$L_{11, i}^{\mathrm{g}}(\mathbf{n})$ is the $(1,1)$ element of the extinction cross-section matrix $\mathbf{L}_{i}^{\mathrm{g}}(\mathbf{n})$.

$a_{1}(\mathbf{n})$ in the second term is the first element of the absorption coefficient vector:

$$
a_{1}(\mathbf{n})=a_{1}^{\mathrm{p}}(\mathbf{n})+a_{1}^{\mathrm{g}}(\mathbf{n}) .
$$

Since gaseous absorption is equal to gaseous extinction,

$$
a_{1}^{\mathrm{g}}(\mathbf{n})=K_{11}^{\mathrm{g}}(\mathbf{n}) .
$$

The particle absorption coefficient $a_{1}^{\mathrm{p}}(\mathbf{n})$ can be expressed as

$$
a_{1}^{\mathrm{p}}(\mathbf{n})=n^{\mathrm{p}} b_{1}^{\mathrm{p}}(\mathbf{n}) .
$$

$b_{1}^{\mathrm{p}}(\mathbf{n})$ is the absorption cross-section of the particles and is calculated as

$$
b_{1}^{\mathrm{p}}(\mathbf{n})=K_{11}^{\mathrm{p}}(\mathbf{n})-\int_{4 \pi} \mathrm{d} n^{\prime} Z_{11}\left(\mathbf{n}, \mathbf{n}^{\prime}\right) .
$$

$Z_{11}\left(\mathbf{n}, \mathbf{n}^{\prime}\right)$ is the $(1,1)$ element of the phase matrix. $Z_{11}\left(\mathbf{n}, \mathbf{n}^{\prime}\right)$ is also computed using the $T$-matrix algorithm by Mishchenko [9].

Our approach is to solve Eq. (1) iteratively with a first guess radiation field. This field can either be the clear sky radiation field or the extinction and thermal source (ETS) field. By ETS field we mean the radiation field generated by the first two terms of Eq. (1).

Assuming local thermodynamic equilibrium, an analytical solution to Eq. (2) for homogenous layers in the absence of the scattering integral is

$$
I_{i, \theta}=I_{i-1, \theta} \exp \left(-\tau_{\mathrm{ext}}\right)+B(T)\left[1-\exp \left(-\tau_{\mathrm{abs}}\right)\right],
$$

where the opacities $\tau_{\mathrm{ext}}$ and $\tau_{\mathrm{abs}}$ are defined by

$$
\tau_{\text {ext }}=1 / \cos (\theta) \int K_{11}(\mathbf{n}) \mathrm{d} z
$$

and

$$
\tau_{\text {abs }}=1 / \cos (\theta) \int a_{1}(\mathbf{n}) \mathrm{d} z,
$$

where $\theta$ is the viewing angle and $i$ denotes the level. The factor $1 / \cos (\theta)$ follows from the plane parallel geometry. By homogenous layers we mean that each layer has a representative extinction 
coefficient, absorption coefficient and temperature. The representative value of each parameter is calculated by taking the mean of the values at adjacent levels. When considering the clear sky case, $\tau_{\mathrm{ext}}=\tau_{\mathrm{abs}}=\tau$.

Assuming optically thin layers, a linear approximation to Eq. (3) is written as

$$
I_{i, \theta}=I_{i-1, \theta}\left(1-\tau_{\mathrm{ext}}\right)+B(T) \tau_{\mathrm{abs}},
$$

which can be solved by traversing the atmosphere layer by layer in the direction of propagation starting with appropriate boundary conditions.

In order for the linear approximation to be valid each layer should be optically thin which is not always the case. Opacity can take large values if the pathlength is large as it is the case when $\theta$ is close to $90^{\circ}$. Here an opacity limit is defined for which the linear approximation is valid within the required accuracy. If the opacity of a layer is higher than this limiting value, the layer is divided further into sub-layers until the opacity is below the limiting value. For $\theta<90^{\circ}$, the iteration starts with the intensity array initialized with the cosmic background radiation, corresponding to a brightness temperature of $2.735 \mathrm{~K}$. The iteration is performed from one layer to the next storing the intensity value for each $\theta$. Upon reaching a layer where clouds are included, extinction as well as emission due to particles are also taken into account. At the ground, the effect of ground reflection is modeled as

$$
I_{0, \theta^{\prime}}=I_{0, \theta}(1-\sigma)+\sigma B\left(T_{\mathrm{G}}\right),
$$

where $\sigma$ is the ground emissivity, $I_{0, \theta^{\prime}}$ is the intensity after reflection, $I_{0, \theta}$ is the intensity before reflection and $T_{\mathrm{G}}$ is the ground temperature. After reflection, the radiation is propagated along the angle $\theta^{\prime}=180^{\circ}-\theta$ and the intensity values are stored for $\theta^{\prime}$. Once the iteration is completed the intensity reaching a point from all directions is obtained. This is essential for the evaluation of the scattering integral. The scattering source term which is computed using this radiation field as the first guess field is used to integrate the full RT equation. The resulting radiation field is used to generate a new radiation field and the iteration is repeated until the solution converges.

\section{Simulation setup}

\subsection{Observation frequencies}

The study is done for three conditions of the atmosphere, the clear sky and two cloudy sky scenarios. For the clear sky case, the frequencies considered are 182, 60 and $90 \mathrm{GHz}$ which have distinctly different features as far as gaseous absorption is concerned. The frequency $182 \mathrm{GHz}$ is at the wing of a strong water vapor absorption line leading to high absorption in the troposphere. The frequency $60 \mathrm{GHz}$ is inside a strong oxygen band and has absorption even at stratospheric levels, and $90 \mathrm{GHz}$ is a window channel which has significant contribution from the surface.

In the case where the effect of clouds is to be taken into account, the frequencies considered are those chosen for the proposed CIWSIR sensor. The CIWSIR channels are $183.31 \pm 1.47,183.31 \pm$ $2.85,183.31 \pm 4.50,325.15 \pm 1.50,325.15 \pm 3.18,325.15 \pm 5.94,448.00 \pm 1.44,448.00 \pm 3.00,448.00 \pm$ $7.20,682.95 \pm 6.00$ and $874.38 \pm 6.00 \mathrm{GHz}$. Presented here are the calculations for 325.15, 462.64, 
682.95 and $874.38 \mathrm{GHz}$. Channel $462.64 \mathrm{GHz}$ is not a part of the CIWSIR but has very similar properties to channel $448 \mathrm{GHz}$ (close in frequency, similar clear sky weighting function).

\subsection{Reference atmosphere and absorption calculation}

The atmospheric scenario studied is mid-latitude winter [10] and the species considered are water vapor, oxygen and nitrogen. The gaseous absorption coefficients are calculated using the RT model ARTS [11]. The absorption coefficients are calculated according to Rosenkranz [12] for water vapor, Rosenkranz [13] for oxygen and Rosenkranz [13] for nitrogen. A recent comparison with the CIMSS-MWLBL model [14], which was used as reference model for ITWG RT model intercomparison [15], shows that the brightness temperatures simulated by ARTS agree with this model with a standard deviation of 0.05 and $0.02 \mathrm{~K}$ for $54.4 \mathrm{GHz}$ (AMSU-06) and $57.290344 \pm 0.217 \mathrm{GHz}$ (AMSU-10), respectively, and $0.15 \mathrm{~K}$ for $57.290344 \pm 0.3222 \pm 0.0045 \mathrm{GHz}$ (AMSU-14) and $0.16 \mathrm{~K}$ for $183.31 \pm 1.0 \mathrm{GHz}$ (AMSU-18). In the present study surface emissivity and temperature are set to 0.65 and $272 \mathrm{~K}$, respectively.

\subsection{Cloud assumptions}

The effect of one strong cirrus cloud and one rather weak cirrus cloud is investigated to explain the effect of scattering. For the strong cirrus cloud, there are larger ice particles at higher temperatures whereas for the weak cirrus cloud there are smaller particles at much lower temperatures. The ice mass content (IMC) is also much larger in the strong cirrus compared to the weak one. According to classifications of cirrus cloud, these two cases of strong and weak cirrus fall more or less under the category of warm and cold cirrus, respectively [16]. It is assumed that all ice crystals are spherical in shape with the same radius and that the cloud is homogenous in the horizontal as well as vertical. Although the assumption of mono-dispersed spherical particles is far from the realistic case, this study is more concerned with our approach to develop an RT model and it is reasonable to start with such a simple assumption. Nevertheless, the model can be extended to incorporate more realistic size-shape distributions of cirrus clouds.

In the case of strong cirrus, the radius of the spherical particles is taken as $100 \mu \mathrm{m}$, and the IMC is $0.02 \mathrm{~g} / \mathrm{m}^{3}$ whereas they are $50 \mu \mathrm{m}$ and $0.003 \mathrm{~g} / \mathrm{m}^{3}$, respectively, for the weak cirrus. Temperature of the ice particles are 250 and $223 \mathrm{~K}$, respectively, for the strong and the weak cases. The scatter plots between IMC, temperature and median-mass diameter by Evans et al. [17] and the relation between temperature and effective radius by Kinne et al. [18] for the FIRE-I campaign suggest that the values considered here can roughly represent cirrus cloud conditions. Since we have mono-dispersed spherical particles the median-mass diameter as well as effective diameter is same as the diameter of the sphere. The strong cloud is $2.5 \mathrm{~km}$ thick with its base height at $7.75 \mathrm{~km}$ and the weak one is $1.5 \mathrm{~km}$ thick with its base height at $10.75 \mathrm{~km}$. The bulk density of ice is taken to be $0.91 \mathrm{~g} / \mathrm{cm}^{3}$.

\subsection{Single scattering properties}

The single scattering properties of ice particles are calculated using T-matrix code for non-spherical particles in a fixed orientation by Mishchenko [9]. Since the particles considered are assumed to be 

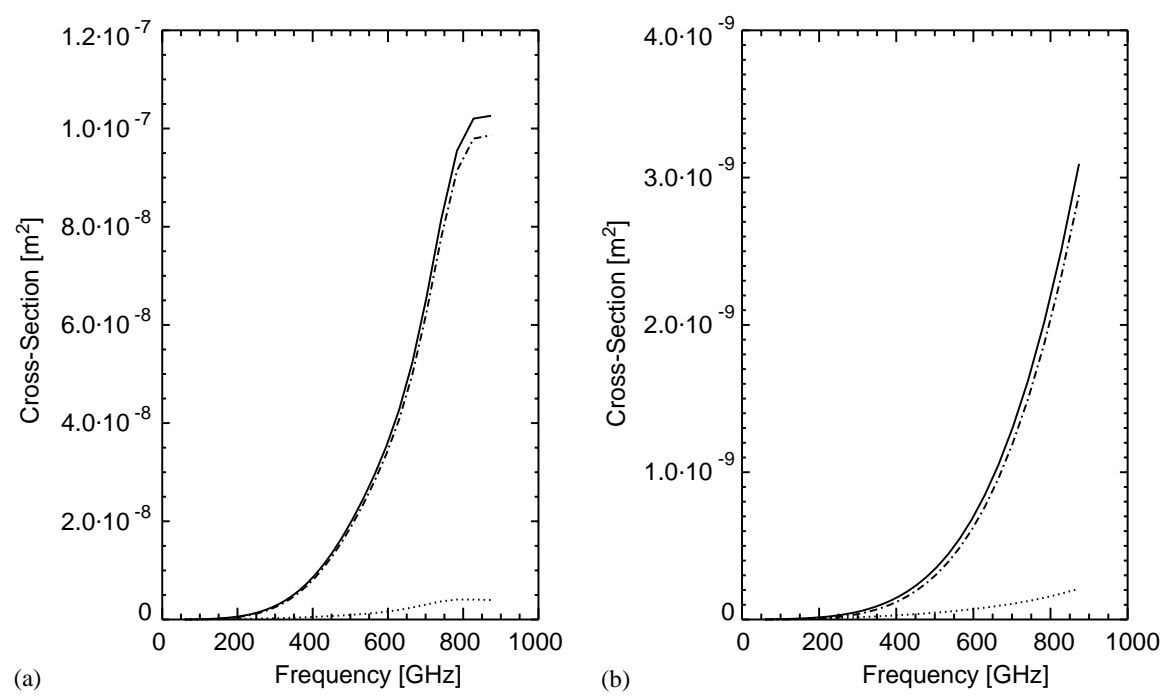

Fig. 1. The extinction, scattering and absorption cross-section for (a) strong cirrus (radius of the spherical ice particle $=100 \mu \mathrm{m}$, temperature $=250 \mathrm{~K}$ ) and (b) weak cirrus (radius of the spherical ice particle $=50 \mu \mathrm{m}$, temperature $=223 \mathrm{~K}$ ) in the frequency range of $60-900 \mathrm{GHz}$. The solid line is the extinction cross-section, the dashed-dotted line is the scattering cross-section and the dotted line is the absorption cross-section.

spherical, the single scattering properties are independent of the orientation. The results of this code completely agree with Mie theory in this frequency range. The program requires as input the equal volume sphere radius of the particle, the frequency under consideration and the corresponding refractive index of ice. The refractive indices as a function of temperature are calculated using the program provided by Warren [19]. The output of the $T$-matrix program gives the scattering amplitude matrices and the scattering efficiency matrices. The phase matrices and the cross-sections are calculated as in Section 2. The behavior of extinction, absorption and scattering cross-sections in this frequency range is shown in Fig. 1. It is seen that for spherical ice particles with radius $50 \mu \mathrm{m}$ and temperature $223 \mathrm{~K}$, the extinction is about one order of magnitude less than that for spheres with radius $100 \mu \mathrm{m}$ and temperature $250 \mathrm{~K}$. For both these cases, the contribution of absorption to the extinction is very small compared to scattering for the frequencies under consideration.

\section{Results and discussion}

\subsection{Clear sky case}

In this section the radiation field inside the atmosphere simulated by the plane parallel model is compared with the spherical model ARTS at 182, 60 and $90 \mathrm{GHz}$. The plane parallel model also has a linear approximation to the solution of the radiative transfer equation. So this section is mainly to understand and compare the effect of these two approximations. The radiances are transformed to equivalent brightness temperatures according to Planck's law. 

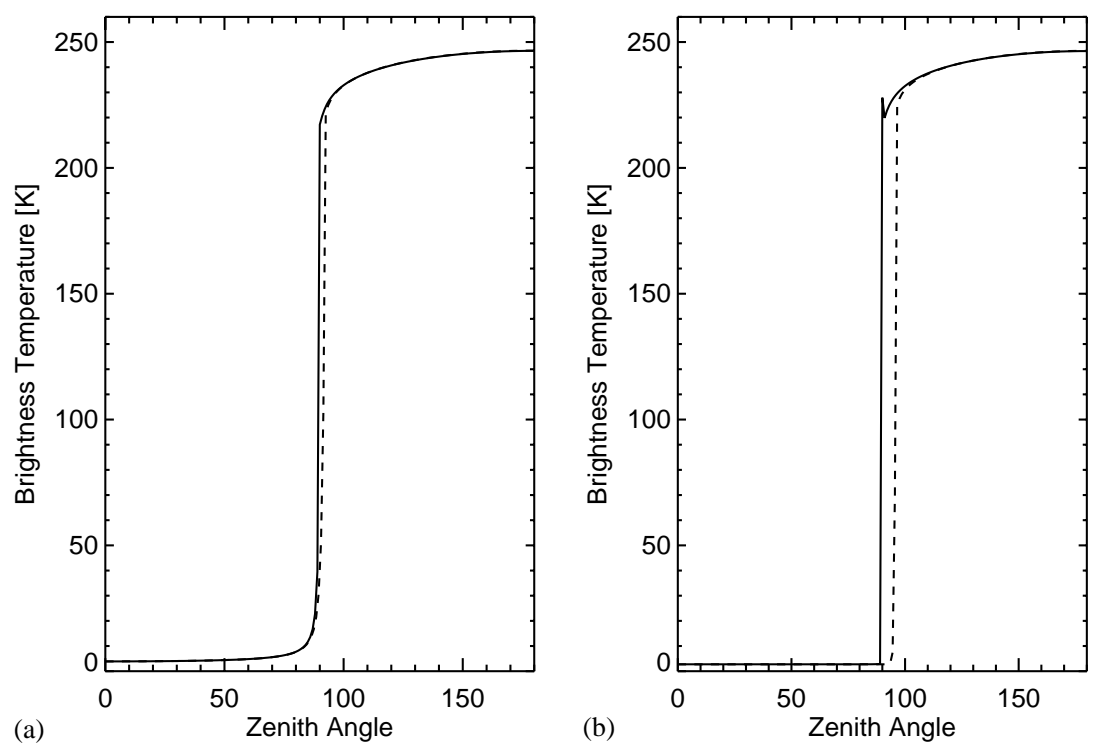

Fig. 2. Comparison of the radiation field at (a) $15 \mathrm{~km}$ and (b) $35 \mathrm{~km}$ of the atmosphere at $182 \mathrm{GHz}$. The solid line is for the plane parallel model and the dashed one is for the spherical model.

Fig. 2 shows the variation of brightness temperature along different viewing angles at 15 and $35 \mathrm{~km}$ simulated by the plane parallel model compared with ARTS at $182 \mathrm{GHz}$. As we look along shallower angles, optical depth being larger, saturation is reached at higher levels which explains the decrease in brightness temperature towards shallower angles. At $90^{\circ}$ the optical depth being infinite, saturation is reached at the same level so that what is seen is the Planck emission corresponding to the temperature of that layer. This explains the peak at $90^{\circ}$ at altitudes corresponding to stratospheric regions. This is a consequence of the plane parallel approximation whereas in the case of spherical geometry optical depth being finite at $90^{\circ}$ this peak is not seen.

Fig. 3 describes the behavior of the same scenario at $60 \mathrm{GHz}$. As against $182 \mathrm{GHz}$, it is seen that at the lower altitude the radiation field is almost identical in all viewing directions. Going further upwards to the stratosphere, it is seen that the emission from top layers has decreased considerably which lowers the brightness temperature in the up-looking angles. Similar to the $182 \mathrm{GHz}$ case, at $90^{\circ}$ the Planck emission corresponding to the temperature of the stratosphere is seen.

Since $90 \mathrm{GHz}$ is a window channel, it shows almost the same behavior at different altitudes. So it is decided to simulate the effect of ground emissivity on the brightness temperature at this frequency. This is shown in Fig. 4, where the effect of different emissivity values on the radiation field at $90 \mathrm{GHz}$ is compared. When the emissivity is 1 , the effect is predominantly from the ground emission, so that the brightness temperature stays constant at all viewing angles $>90^{\circ}$. When the emissivity is zero, the major contribution is from atmospheric emission. Hence towards shallower angles the brightness temperature increases as optical depth increases. When the emissivity is at an intermediate value it is seen that the effect depends on the compensation between the extinction and emission terms in the transfer equation. 

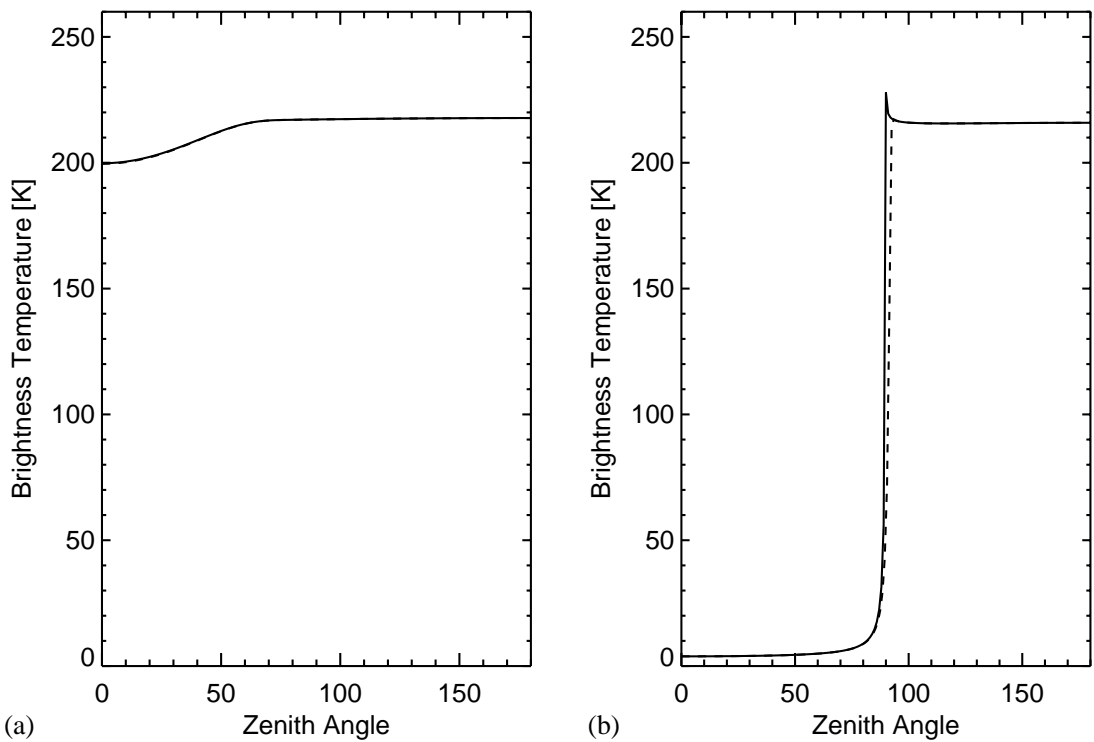

Fig. 3. Comparison of the radiation field at (a) $15 \mathrm{~km}$ and (b) $35 \mathrm{~km}$ of the atmosphere at $60 \mathrm{GHz}$. The solid line is for the plane parallel model and the dashed one is for the spherical model.
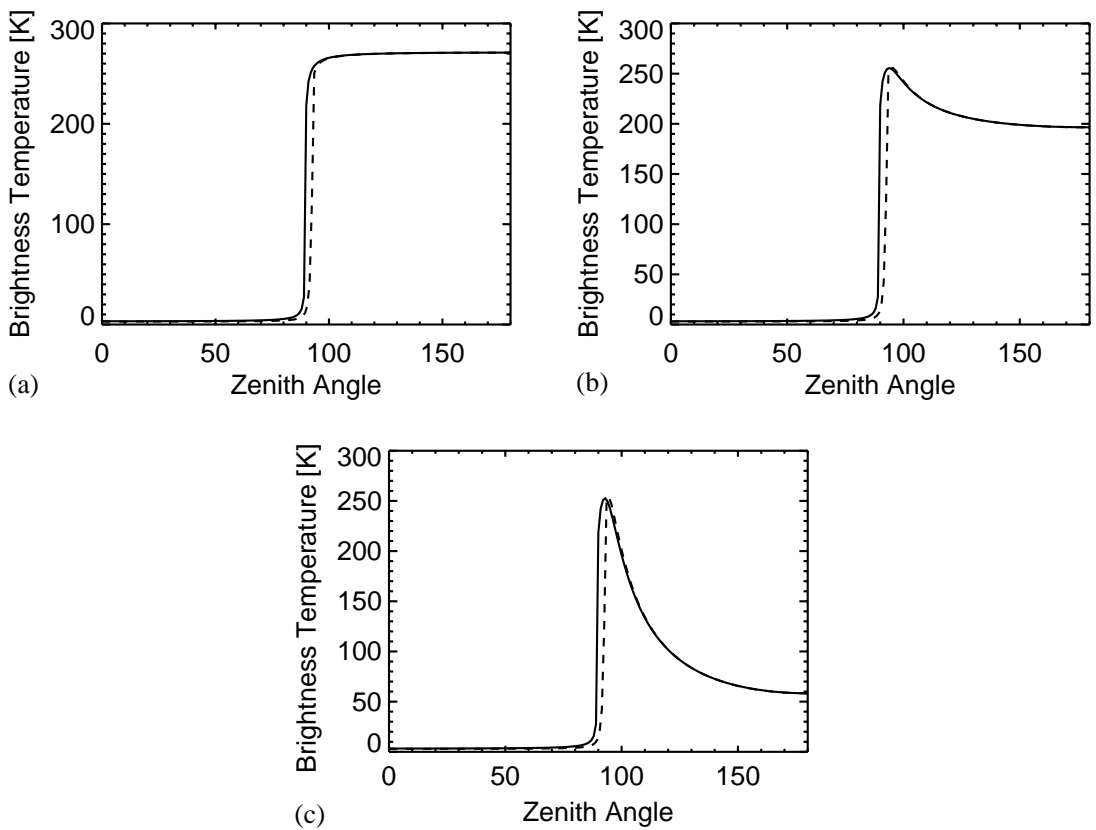

Fig. 4. Comparison of the radiation field at $15 \mathrm{~km}$ inside the atmosphere for different surface emissivities at $90 \mathrm{GHz}$. The solid line is for the plane parallel model and the dashed one is for the spherical model. Emissivity values are (a) 1.0, (b) 0.65 and (c) 0.0 . 

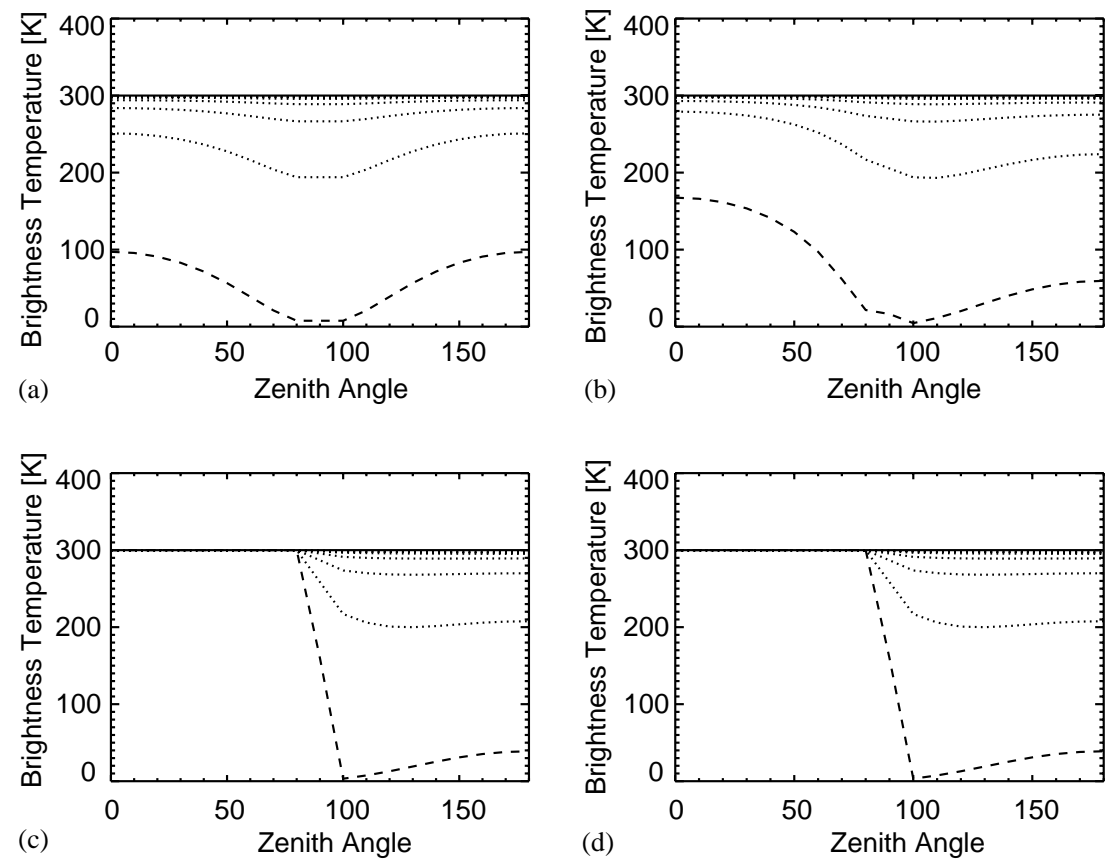

Fig. 5. The convergence of the multiply scattered field to the clear sky field in the case of an atmosphere with no absorption and no surface emission at $874 \mathrm{GHz}$ for the strong cirrus cloud case (radius of the spherical ice particle $=100 \mu \mathrm{m}$, temperature $=250 \mathrm{~K}, \mathrm{IMC}=0.02 \mathrm{~g} / \mathrm{m}^{3}$, thickness $=2.5 \mathrm{~km}$ ) at (a) $4 \mathrm{~km}$, (b) $6 \mathrm{~km}$, (c) $25 \mathrm{~km}$ and (d) $80 \mathrm{~km}$. The solid line represents the clear sky field, the dashed line the ETS field and the dotted lines are the first 30 multiply scattered fields in intervals of 5.

\subsection{Scattering convergence test}

The scattering convergence test is for determining the consistency of the radiative transfer calculations done here. For this test, the cosmic background temperature is set to a hypothetical value of $300 \mathrm{~K}$. The absorption coefficients for gases as well as for particles and the ground emissivity are set to zero. In such an atmospheric scenario, for the clear sky case, the calculation of the radiation field propagating in all directions should give the same value of $300 \mathrm{~K}$ at all altitudes of the atmosphere since there are no sources or sinks involved. The only factor which changes the field in a cloudy case is the scattering due to cloud particles. The physics of this problem suggests that after a considerable number of iterations the field should converge to the clear sky case. This is because scattering only does a redistribution of energy in different directions. Fig. 5 shows the convergence test for the strong cloud case. The solid line represents the clear sky case, the dashed one is the first guess field, the ETS field. The dotted lines are the first 30 iterations in an interval of five. The case presented here is the worst case for the convergence (high IMC, single scattering albedo 1, high frequency). In all realistic simulations convergence was reached in less than 15 iterations.

The convergence was also successfully tested when the first guess field is set to an arbitrary value of $500 \mathrm{~K}$ in all directions. This suggests that it is not necessary that always the first guess field should be the ETS field, even clear sky field can be a suitable candidate. 

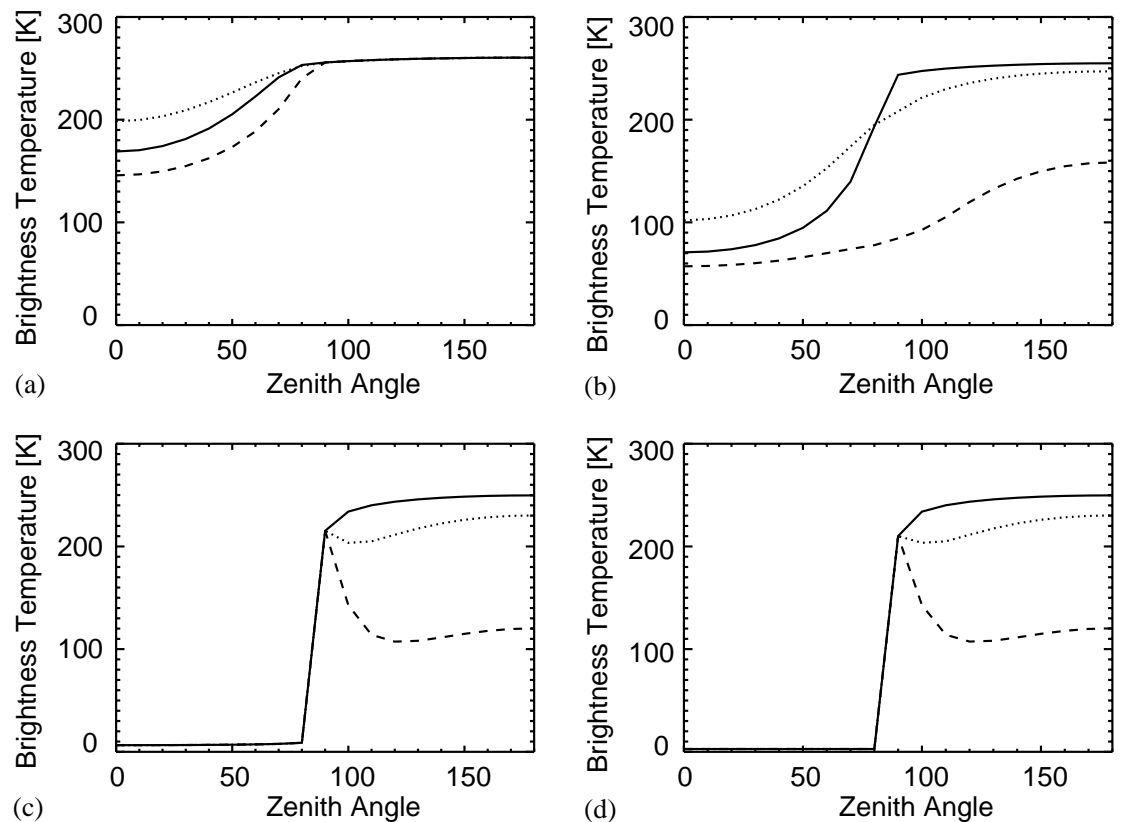

Fig. 6. The radiation field inside the atmosphere for a strong cirrus case (radius of the spherical ice particle $=100 \mu \mathrm{m}$, temperature $=250 \mathrm{~K}, \mathrm{IMC}=0.02 \mathrm{~g} / \mathrm{m}^{3}$, thickness $=2.5 \mathrm{~km}$ ) at $874 \mathrm{GHz}$ at (a) $4 \mathrm{~km}$, (b) $6 \mathrm{~km}$, (c) $25 \mathrm{~km}$ and (d) $80 \mathrm{~km}$ of the atmosphere. The solid line is the clear sky field, the dashed one is the ETS field and the dotted line is the multiply scattered field after convergence.

\subsection{Cloud case}

With the confidence gained from the clear sky comparison and the scattering convergence test, we proceed to the cloudy condition. As an illustration of the process the case of strong cirrus at $874 \mathrm{GHz}$ is considered. Fig. 6 shows the angular variation of brightness temperature for the clear sky case and the cloudy case at different heights in the atmosphere. Fig. 6a is for the case below the cloud, Fig. $6 \mathrm{~b}$ is for inside the cloud and Figs. $6 \mathrm{c}$ and $\mathrm{d}$ are for above the cloud. The clear sky field shown by solid line remains almost constant for the downlooking angles at all altitudes. In the up-looking case at lower altitudes emission from the top is seen whereas at higher altitudes only cosmic background is seen.

The dashed line represents the ETS field. Since scattering is many orders of magnitude higher than absorption for cloud particles, the field is affected negligibly by cloud emission. The dotted line shows the scattered field computed by iterating Eq. (2) with the ETS field as the first guess field. The scattered field converges after about 15 iterations. For downlooking angles viewing from above the cloud $(25$ and $80 \mathrm{~km}$ ) scattering decreases the brightness temperature compared to the clear sky case. For uplooking cases looking from below the cloud $(4 \mathrm{~km})$ scattering increases the brightness temperature. Of particular interest is the field inside the cloud at $6 \mathrm{~km}$ where we see how scattering actually does a redistribution of radiation by comparing the scattered field with the clear sky field. Another noticeable point is that the scattered field is closer to the clear sky field implying that an iteration procedure with the clear sky field as the first guess can yield the result with a fewer 

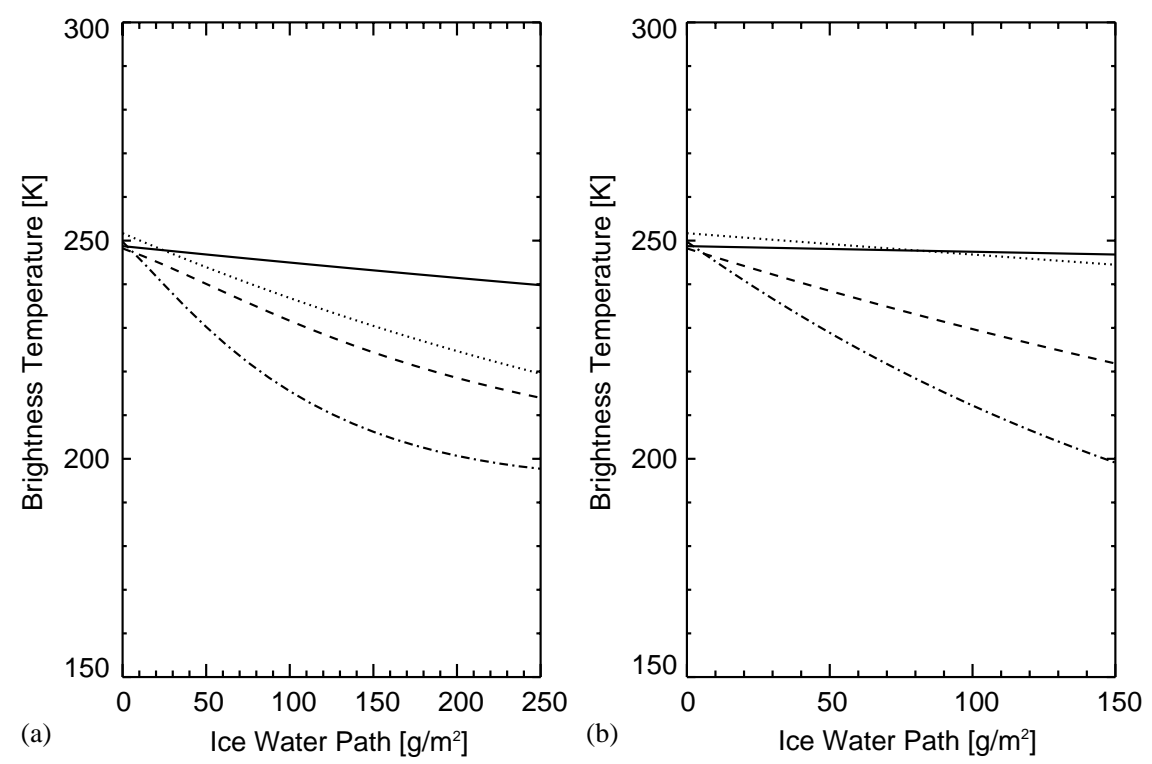

Fig. 7. Effect of IWP on brightness temperature for (a) strong cirrus (radius of the spherical ice particle $=100 \mu \mathrm{m}$, temperature $=250 \mathrm{~K}$ ) and $(\mathrm{b})$ weak cirrus (radius of the spherical ice particle $=50 \mu \mathrm{m}$, temperature $=223 \mathrm{~K}$ ) for Nadir view. The solid line is for $325 \mathrm{GHz}$, dotted line is for $463 \mathrm{GHz}$, dashed line is for $683 \mathrm{GHz}$ and dashed-dotted line is for $874 \mathrm{GHz}$.

number of iterations. This is also attempted and is found that the same field is generated with six iterations. Since the resulting field is independent of the first guess field one can always try to find better choices for the initial guess field.

The sub-mm channels located in the strong water vapor absorption region offer a number of advantages as far as ice clouds are concerned [20]. CIWSIR is aiming at exploiting the property of sub-mm channel that the brightness temperature depression is approximately proportional to the integrated ice mass. Using the information from the ratios of brightness temperature depressions at adjacent frequencies to determine the characteristic size of the ice particles, the measured brightness temperature can be converted to cirrus IWP [20]. Fig. 7 shows the upwelling radiance at the top of the atmosphere when looking nadir at different sub-mm wavelengths for the two cloud cases discussed above. Only the IMC is varied, all other parameters are kept the same. The horizontal axis shows the IWP. The vertical axis shows the radiance in units of Planck brightness temperature. The brightness temperature depression due to the presence of ice particles is closely related to the IWP. For a fixed IWP, there is a greater brightness temperature change for larger ice particles or higher measurement frequencies. Furthermore, over a large range of IWP and frequency the RT occurs in the linear regime, i.e., the brightness temperature depression is proportional to the IWP. Only at very high frequencies or high IWP does the behavior become non-linear.

\section{Conclusions}

We have tried to implement two approximations in this approach. One is a linear approximation of the exponential term in the radiative transfer equation and the other is plane parallel approximation 
for representing earth's geometry. Both these approximations are tested against a spherical model with exponential solution for the clear sky case. The linear approximation seems to yield good results if the limiting value is set for the optical depth and then split the layers until optical depth becomes smaller than the limiting value. With the plane parallel approximation of the atmosphere, our results indicate that a plane parallel atmosphere can give comparable results to the spherical atmosphere particularly in the troposphere. But at stratospheric altitudes there are problems close to $90^{\circ}$ viewing angles as expected.

For the cloudy case, before proceeding to a real cloud scenario the results of a convergence test are presented, which gives us confidence to continue working with the model. Since the test case represents one of the worst cases, it required around 30 iterations whereas in a realistic case only fewer iterations are required. Furthermore, a smart choice of the initial guess field can considerably accelerate convergence.

The effect of scattering depends highly on the frequency of the radiation and on the particle size and shape. For the cirrus cloud cases those we have considered, the scattering and extinction cross-section increases with frequency. This explains why $874 \mathrm{GHz}$ shows the highest brightness temperature change in both the cloud cases followed in order by 683,463 and $325 \mathrm{GHz}$. The simulated brightness temperatures are mostly in the linear regime which makes them particularly suitable for getting information about IWP and particle size.

\section{Acknowledgements}

We thank Michael Mishchenko and Stephen Warren for making available the $T$-matrix program and the refractive index program respectively. We also thank Frank Evans for discussions and information on the cloud scenarios. Furthermore, the authors wish to acknowledge funding through the German research projects SMILES (DLR 50EE9815) and UTH-MOS (GSF 07ATC04).

\section{References}

[1] Diak GR, Kim D, Whipple MS, Wu X. Preparing for the AMSU. Bull Am Meteorol Soc 1992;73:1971-84.

[2] Kramer HJ. Observation of the earth and its environment: survey of missions and sensors. Berlin, Heidelberg, New York: Springer, 1996.

[3] Miao J, Heygster G, Kunzi K. Appendix-7: ice cloud and background water vapor by sub-millimeter and very high frequency microwave radiometry. Clouds - a cloud and radiation monitoring satellite: final report to the EU, 2000.

[4] Evans KF, Evans AH, Nolt IG, Marshall BT. The Prospect for remote sensing of cirrus clouds with a submillimeter-wave spectrometer. J Appl Meteorol 1999;38:514-25.

[5] Evans, Franklin K, Stephens GL. A new polarized atmospheric radiative transfer model. J Quant Spectrosc Radiat Transfer 1991;46:413-23.

[6] Czekala H, Simmer C. Microwave radiative transfer with nonspherical precipitating hydrometeors. J Quant Spectrosc Radiat Transfer 1998;60:365-74.

[7] Mishchenko MI, Hovenier JW, Travis LD. Light scattering by non-spherical particle: theory, measurement and applications. London: Academic Press, 2000.

[8] Goody RM, Yung YL. Atmospheric radiation - theoretical basis. New York: Oxford University Press, 1995.

[9] Mishchenko MI. Calculation of the amplitude matrix for a nonspherical particle in a fixed orientation. Appl Opt 2000;39:1026-31.

[10] Claugh SA, Kneizys FX, Shettle EP, Anderson GP. Atmospheric radiance and transmittance: FASCOD2. In: Proceedings of the Sixth Conference on Atmospheric Radiation, American Meteorological Society, 1986. 
[11] Buehler S, Eriksson P. www.sat.uni-bremen.de/ARTS.

[12] Rosenkranz PW. Water-vapour microwave continuum absorption: a comparison of measurements and models. Radio Sci 1998;33:919-28.

[13] Rosenkranz PW. Absorption of microwaves by atmospheric gases. In: Janssen MA, editor. Atmospheric remote sensing by microwave radiometry. London: Wiley, 1993. p. 37-90.

[14] Rosenkranz PW. A rapid atmospheric transmittance algorithm for microwave sounding channels. IEEE Trans Geosci Remote Sensing 1995;33:1135-40.

[15] Garand L, Turner DS, Larocque M, Bates J, Boukabara S, Brunel P, Chevallier F, Deblonde G, Engelen R, Hollingshead M, Jackson D, Jedlovec G, Joiner J, Kleespies T, McKague DS, McMillin L, Moncet JL, Pardo JR, Rayer PJ, Salathe E, Saunders R, Scott NA, Van Delst P, Woolf H. Radiance and Jacobian intercomparison of radiative transfer models applied to HIRS and AMSU channels. J Geophys Res 2001;106:24017-31.

[16] Heymsfield AJ, Platt CMR. A parameterization of the particle size spectrum of ice clouds in terms of the ambient temperature and the ice water content. J Atmos Sci 1984;41:846-55.

[17] Evans FK, Walter SJ, Heymsfield AJ, McFarquhar GM. The submillimeter-wave cloud ice radiometer: simulations of retriveal algorithm performance. J Geophys Res 2001, in press.

[18] Kinne S, Akerman TP, Shiobara M, Uchiyama A, Heymsfield AJ, Miloshevich L, Wendell J, Eloranta EW, Purgold C, Bergstrom RW. Cirrus cloud radiative and microphysical properties from ground observations and in situ measurements during FIRE 1991 and their application to exhibit problems in cirrus solar radiative transfer modeling. J Atmos Sci 1995;54:2320-44.

[19] Stephen G. Warren, Optical Constants of ice from the ultraviolet to the microwave. Appl Opt 2000;23:1206-25.

[20] Evans, Franklin K, Stephens GL. Microwave radiative transfer through clouds composed of realistically shaped ice crystals. Part II: remote sensing of ice clouds. J Atmos Sci 1995;52:2058-72. 